\title{
Roda de conversa em um ambulatório público: o papel da atenção primária na educação popular em saúde
}

\author{
Conversation circle in a public clinic: the role of primary care in popular health education \\ Círculo de conversación en una clínica pública: el papel de la atención primaria en la educación
} sanitaria popular

Recebido: 30/09/2021 | Revisado: 09/10/2021 | Aceito: 16/10/2021 | Publicado: 20/10/2021

\author{
Márcia Farsura de Oliveira \\ ORCID: https://orcid.org/0000-0001-8462-0431 \\ Faculdade Dinâmica do Vale do Piranga, Brasil \\ E-mail: mmfarsura@yahoo.com.br \\ Pollyanna Álvaro Ferreira Spósito \\ ORCID: https://orcid.org/0000-0003-0875-6227 \\ Faculdade Dinâmica do Vale do Piranga, Brasil \\ E-mail: pollysposito@gmail.com \\ Caroline Silva de Araujo Lima \\ ORCID: https://orcid.org/0000-0003-2537-292X \\ Faculdade Dinâmica do Vale do Piranga, Brasil \\ E-mail: carolinearaujo689@gmail.com \\ Marli do Carmo Cupertino \\ ORCID: https://orcid.org/0000-0001-5790-0622 \\ Faculdade Dinâmica do Vale do Piranga, Brasil \\ E-mail: marlicuppertino@gmail.com
}

\begin{abstract}
Resumo
A Atenção Primária à Saúde (APS), por meio do atendimento humanizado, resgata os vínculos de compromisso entre os serviços de saúde, os profissionais e a população, de forma mais eficaz, por meio da educação popular em saúde (EPS). Objetivou-se realizar, na sala de espera de um ambulatório público - Ambulatório Sette de Barros (ASB) uma roda de conversa (RCO), e descrever o papel da APS na EPS, como fonte de informações acessíveis e seguras. Após aprovação Ética, número do parecer 2.925.286, foi aplicado questionário impresso para 378 pessoas - número definido por cálculo de amostra finita -, nos meses de setembro e novembro de 2018. Após a pesquisa identificar o interesse em participar das ROC por 79,62\% dos entrevistados, foram realizadas três ROC, no período compreendido entre março a abril de 2019, com subsequente avaliação do evento dialógico. A pesquisa identificou como principais pontos positivos o fácil entendimento e as trocas de experiências e como aspecto negativo, referido pela maioria, o tempo muito curto. A APS desempenha um papel crucial no fornecimento de informações aos usuários sendo a principal fonte de informações seguras e de fácil acesso. Por essa razão, a ferramenta de rodas de conversa promove EPS, sendo um importante integrador do ensino-serviço-pesquisa. A população necessita de mais momentos de discussão com os profissionais de saúde para demonstrarem as lacunas de conhecimento e viabilizarem proposta de integração ensinoserviço, favorecendo a satisfação e empoderamento dos usuários das ações em saúde prestadas pelo ASB.
\end{abstract}

Palavras-chave: Atenção primária à saúde; Educação médica; Política pública.

\begin{abstract}
Primary Health Care (APS), through humanized care, rescues the bonds of commitment between health services, professionals and the population, more effectively, through popular health education (EPS). The objective was to carry out, in the waiting room of a public clinic - Sette de Barros Ambulatory (ASB), a conversation circle (RCO), and describe the role of APS in EPS, as a source of accessible and safe information. After Ethics approval, opinion number $2,925,286$, a printed questionnaire was applied to 378 people - number defined by finite sample calculation -, in September and November 2018. After the research identified the interest in participating in the ROC by $79,62 \%$ of respondents, three ROCs were carried out, in the period between March and April 2019, with subsequent evaluation of the dialogic event. The research identified as the main positive points the easy understanding and the exchange of experiences, and as a negative aspect, mentioned by most, the very short time. APS plays a crucial role in providing information to users and is the main source of secure and easily accessible information. For this reason, the conversation wheel tool promotes EPS, being an important integrator of teaching-service-research. The population needs more moments of discussion with health professionals to demonstrate the knowledge gaps and enable a proposal for teachingservice integration, favoring the satisfaction and empowerment of users of the health actions provided by the ASB.
\end{abstract}


Keywords: Primary health care; Education, medical; Public policy.

\section{Resumen}

La Atención Primaria de Salud (APS), a través de la atención humanizada, rescata los lazos de compromiso entre los servicios de salud, los profesionales y la población, de manera más efectiva, a través de la Educación Popular en Salud (EPS). El objetivo fue realizar, en la sala de espera de una clínica pública - Ambulatorio Sette de Barros (ASB), un círculo de conversación (RCO), y describir el papel de la APS en EPS, como fuente de información accesible y segura. Luego de la aprobación de Ética, opinión número 2,925,286, se aplicó un cuestionario impreso a 378 personas - número definido por cálculo de muestra finita -, en septiembre y noviembre de 2018. Luego de que la investigación identificó el interés en participar en la República de China por 79, 62\% de los encuestados, Se realizaron tres ROC, en el período comprendido entre marzo y abril de 2019, con posterior evaluación del evento dialógico. La investigación identificó como principales puntos positivos la fácil comprensión y el intercambio de experiencias, y como aspecto negativo, mencionado por la mayoría, el muy corto tiempo. APS juega un papel crucial en el suministro de información a los usuarios y es la principal fuente de información segura y de fácil acceso. Por ello, la herramienta rueda de conversación promueve la EPS, siendo un importante integrador de la docencia-servicio-investigación. La población necesita más momentos de discusión con los profesionales de la salud para demostrar las brechas de conocimiento y posibilitar una propuesta de integración docente-servicio, favoreciendo la satisfacción y empoderamiento de los usuarios de las acciones de salud que brinda la ASB.

Palabras clave: Atención primaria de salud; Educación médica; Política pública.

\section{Introdução}

A política pública, de saúde brasileira, é um campo no qual se integram outras políticas públicas. Tais políticas devem apresentar na formação da agenda/pauta, fatores que possibilitem que os direitos dos cidadãos sejam garantidos, de forma justa e com equidade. Direitos como Educação Permanente em Saúde permitem a tomada de decisões na atenção em saúde, com conduta terapêutica estabelecida de forma conjunta com o paciente, a partir de autonomia intelectual possibilitada ao mesmo (Ferreira et al., 2019).

No Brasil, o direto à saúde é garantido por meio do Sistema Único de Saúde (SUS) que deve ser universal, integral e gratuito (Ferreira et al., 2019). A formulação de políticas públicas, baseadas na promoção da saúde e prevenção de agravos, causou algumas modificações no processo de trabalho em saúde. As principais modificações possuem enfoque na bioética, valorização do trabalho em equipe - interdisciplinar e multiprofissional -, e desenvolvimento de habilidades de comunicação para uma boa formação da relação médico-paciente (Brasil, 2014).

Em atendimento à necessidade de formação de recursos humanos para o Sistema Único de Saúde (SUS), foram promulgadas as Diretrizes Curriculares Nacionais para o Curso de Graduação em Medicina de 2014 (DCNs), que trazem orientações pedagógicas, além de habilidades e competências a serem adquiridas pelo graduando de tal curso da área da saúde (Brasil, 2014). Tais diretrizes foram publicadas após a lei de criação do Programa Mais Médicos - Lei $n^{0} 12.871$ de 2013 -, cujo foco principal consiste na formação médica voltada para o SUS, para que as desigualdades de acesso à saúde, sejam reduzidas ou inexistentes, no território brasileiro. Os principais preceitos das DCNs valorizam a ideia de que o ensino-serviço ocorra desde o início do curso, existindo contato do discente com a comunidade para identificação dos agravos mais prevalentes na comunidade e priorização de ações para as necessidades de saúde (Pellegrini Filho, Buss, \& Esperidião, 2013).

A utilização de metodologias ativas de ensino-aprendizagem, como a realização de atividades acadêmicas em cenários práticos, envolvendo acompanhamento de atendimentos médicos e educação popular em saúde, gera ganhos mútuos na interação dos graduandos de Medicina com os usuários dos serviços de saúde (Bordenave, 1999; Adler \& Gallian, 2014; França \& Maknamara, 2019). Tal fato, apresenta um convite a enxergar a teoria como observação atenta do prático, permitindo tomada de consciência e melhoria do aprendizado (Freire, 1999).

A proposta freiriana não é que a contemplação seja com visão de algo que só exista nas ideias, ou que esteja em equilíbrio, mas é aquela que visa a transformação, sem que haja acomodação ou descaso com a situação que se apresenta (Morin, 2003; 
Paro, Ventura \& Silva, 2019). E é de forma semelhante ao que foi proposto por Freire, que devem ser planejadas as estratégias educativas em saúde, visando a confluência de entendimentos teóricos e práticos (Oliveira \& Cota, 2018).

No SUS, a educação em saúde integra duas políticas públicas: (i) a Educação Permanente em Saúde, que consiste no aperfeiçoamento ou capacitação dos profissionais, e (ii) a Educação Popular em Saúde, que certifica que o conjunto de conhecimentos sobre algo ou alguém são obtidos, de forma diversa e, a partir da comunicação, diálogo, trato, contato entre pessoas que convivem (Adler \& Gallian, 2014; Silva \& Scherer, 2020).

A Política Nacional de Educação Popular em Saúde de 2012 objetiva valorizar as ações gerais realizadas pela população, relacionadas ao bem-estar, com soberania popular e controle social, não se restringindo às atividades desenvolvidas pelos Agentes Comunitários de Saúde, mas como parte do processo de trabalho em saúde no SUS, na abordagem individual ou em grupos (Oliveira \& Cota, 2018).

O comprometimento com a prática dos direitos e deveres de uma pessoa, em um Estado, consiste em uma das bases das DCNs (Pellegrini Filho, Buss, \& Esperidião, 2013), as quais conservaram o estabelecimento de vínculos das atividades práticas desenvolvidas pelos graduandos em Medicina com os diagnósticos situacionais de saúde da população e do SUS, afirmando o planejamento de ensino estruturado por aptidões e conhecimentos necessários à profissão, com recurso de metodologias ativas de aprendizagem, em três principais eixos: (i) Atenção à Saúde; (ii) Gestão em Saúde e (iii) Educação em Saúde (Brasil, 2014).

$\mathrm{O}$ eixo atenção é guiado por fundamentos de possibilidade de garantia de direito à saúde, como previsto na Lei 8.080 de 1990, uma das Leis Orgânicas da Saúde (LOS), com equidade e integralidade, com atendimento humanizado e pautado pelas normas da bioética (Adler \& Gallian, 2014; Costa et al., 2018). A gestão deve garantir o fortalecimento dos foros gestores de participação popular, com vigilância das premências locais e da região, quanto ao respeito à vida, às escolhas e determinações, de acordo com as realidades apresentadas pelas situações, às habilidades de comunicação e formação da relação profissionalpaciente e do vínculo interprofissional, à capacidade de coordenar uma ação em saúde, ao incentivo da participação popular e do controle social (Brasil, 2014). A Educação em Saúde tem como escopo, a valorização do trabalho interdisciplinar e multiprofissional, conhecimento técnico-científico aliado à humanização, favorecendo a autonomia e autocuidado do educando - nesse caso, o usuário do SUS (Ferreira et al., 2019).

A metodologia de ensino que emprega a abordagem humanista é aquela com foco no aprendiz, que é o protagonista e o condutor da trilha pedagógica, onde ocorrerá atribuição de novo significado a acontecimentos através da mudança de sua visão de mundo, com consequente alteração integral de caráter e de personalidade (Brasil, 2013). Em grupos, pode ser realizada através de rodas de conversa, compreendida como constituição de momentos mediados por uma pessoa, que normalmente é o profissional de saúde, oportunos para as trocas de conhecimentos a respeito de determinado assunto, numa relação entre iguais, ou seja, dois seres humanos com seus direitos naturais e que colaboram por perceberem vantagem nisso (Oliveira \& Leite, 2011; Nascimento \& Baduy, 2021).

Essa construção de conhecimentos conjunta, estabelecida entre profissionais de saúde e clientes, possibilita a maior autonomia dos sujeitos sobre as decisões quanto às terapêuticas, contribuindo, assim, para a integralidade da atenção, centrada no indivíduo, com sugestões democráticas, oportunizando o controle social e aumentando o compromisso com a cidadania (Bordenave, 1999; Ferreira et al., 2014).

A partir dos dados abordados nos parágrafos anteriores e inicialmente com a participação de discentes do terceiro período da escola de Medicina da Faculdade Dinâmica do Vale do Piranga (FADIP) em um serviço de atenção primária à saúde, este estudo objetivou analisar o resultado da integração das seguintes políticas públicas: (i) o Programa de Saúde da Família criado em 1994 -, e (ii) a Política Nacional de Educação Popular em Saúde no âmbito do Sistema Único de Saúde (PNEPS-SUS, além de políticas de participação e controle social (conselhos, conferências de saúde) e iniciativas de participação popular. Através da realização de avaliação por meio de pesquisa de opinião da clientela atendida no ambulatório Sette de Barros, 
abordando os temas de saúde propostos, optamos pelas escolhas, para a introdução dialógica na roda de conversa. A partir das duas estratégias de saúde da família propôs-se a discussão em rodas de conversa e, após a realização do momento educativo mediado por acadêmicos de Medicina da FADIP utilizando cartilha elaborada a partir do tema eleito pelos usuários, investigar a concepção evidenciada pelos usuários do ASB sobre o momento dialógico educativo.

\section{Metodologia}

Trata-se de um relato de experiência de caráter exploratório, descritivo, com pesquisa de abordagem quali-quantitativa, a qual foi desenvolvida no ASB aberto à participação da comunidade da área de abrangência das Estratégias Saúde da Família (ESF) Sette de Barros I e Sette de Barros II, na cidade de Ponte Nova, Minas Gerais, Brasil, que, de acordo com o DATASUS, o total de pacientes atendidos correspondem a, aproximadamente, 5.562 pessoas (Brasil, 2015).

O estudo foi realizado em duas etapas: i) coleta de dados pelos discentes da FADIP, por meio de um questionário impresso, seguindo um roteiro semi-estruturado com duas perguntas objetivas. Para estabelecer o tamanho da amostra para a aplicação do primeiro questionário realizou-se um cálculo de amostra finita, com nível de confiança de 95\% e margem de erro de $5 \%$, com estimativa de perda de $20 \%$, por não atendimento aos questionários. Nos meses de setembro e novembro de 2018 foram aplicados 378 questionários. A eleição dos clientes adscritos às ESF que responderam ao questionário foi aleatória; ii) análise dos dados e realização das rodas de conversa, onde foi feita uma nova coleta de dados, através de um questionário com sete questões objetivas apresentado subsequentemente ao momento dialógico. Um universo de sessenta pacientes participou das rodas de conversa, com duração de 15 minutos, nos meses de março e abril de 2019, nas tardes de quarta-feira, dia da semana no qual acontecem as atividades práticas dos discentes do terceiro período do curso de Medicina da FADIP no ASB. Os critérios de inclusão foram: pacientes maiores de 18 anos, alfabetizados, que frequentaram o serviço de atenção primária à saúde dos bairros Triângulo Novo, Triângulo Velho e São Judas, e que participaram livremente da pesquisa após assinarem o termo de consentimento livre e esclarecido.

O estudo foi realizado mediante a aprovação do Comitê de Ética em Pesquisa (CEP) da Faculdade Dinâmica do Vale do Piranga, com número de parecer 2.925.286, em atendimento à orientação da Resolução 466/12 do Conselho Nacional de Saúde (CNS) uma vez que a pesquisa envolveu seres humanos.

Após coleta e tabulação dos dados, foi realizada a análise descritiva dos mesmos. As variáveis categóricas foram expressas em frequências absolutas e relativas e as variáveis quantitativas foram expressas como média e desvio padrão. O software utilizado foi o Epi Info versão 3.5.1.

\section{Resultados e Discussão}

As DCNs apoiam a orientação de formação de recursos humanos por meio de ações em cenários práticos, desde o início da graduação, que tenha como eixos norteadores a atenção, gestão e educação em saúde. Para atender às propostas de articulação ensino-serviço, a Intituição de Ensino Superior (IES) incentiva a inserção dos estudantes dos primeiros períodos do curso de Medicina nos serviços de saúde públicos de Ponte Nova e região, incluindo os graduandos do terceiro período, de onde são provenientes os oito acadêmicos que frequentaram o cenário prático do ASB e tiveram oportunidade de participar da pesquisa descrita neste artigo.

Os dados obtidos a partir da análise do primeiro questionário aplicado aos usuários da área de abrangência do ambulatório Sette de Barros, um serviço de Atenção Primária à Saúde (APS), no município de Ponte Nova, Minas Gerais estão representados apresentados na Quadro 1. 
Quadro 1 - Análise do primeiro questionário aplicado aos usuários da área de abrangência do ambulatório Sette De Barros, no município de Ponte Nova, Minas Gerais.

\begin{tabular}{|c|c|}
\hline Frequência de comparecimento ao $\mathrm{ASB}^{\mathrm{a}}$ & $\begin{array}{l}1 \text { vez a cada } 15 \text { dias }-41^{x} \\
1 \text { vez ao mês }-27^{x} \\
1 \text { vez a cada } 3 \text { meses }-53^{x} \\
2 \text { vezes ao ano }-193^{x} \\
1 \text { vez ao ano - } 43^{x} \\
\text { Não sabe }-21^{x}\end{array}$ \\
\hline $\begin{array}{l}\text { Opinião quanto ao interesse em participar de roda de } \\
\text { conversa durante o tempo de espera por consulta no } \\
\text { ASB }\end{array}$ & $\begin{array}{l}\text { Tenho interesse }-301^{x} \\
\text { Não tenho interesse }-77^{x}\end{array}$ \\
\hline
\end{tabular}

Fonte: Autores (2021). ${ }^{\mathrm{a} A S B}$ : ambulatório Sette de Barros. ${ }^{\mathrm{x}}$ Número de respostas dadas pelas pessoas que responderam aos questionários.

Verificou-se que a maioria dos usuários (314), frequenta o serviço de atenção primária até duas vezes por ano, o que significa que provavelmente o cliente que participou do primeiro questionário não participaria da roda de conversa, por causa da frequência de vezes que vai ao ASB. Por isso, o tema proposto para discussão deveria ser representativo, para satisfazer a maioria dos usuários do serviço de saúde, com expectativa de continuidade dos momentos educativos a fim de permitir a mais clientes ouvirem sobre o assunto, com maior abrangência da Educação Popular em Saúde, proposta por esta pesquisa.

Entende-se que o planejamento da atividade pedagógica voltada para a saúde não pode restringir-se ao modelo biomédico - tecnicista - sobre o saber popular, pois, dessa forma, a maneira de apresentação da informação fica centrada no modelo cartesiano de descrição da doença e não valoriza as questões sociais e a integralidade do enfermo (Echer, 2005; Starfield, 2002). E levando em conta, ainda, que somente alterações na técnica de ensino não são suficientes para alcançar as habilidades humanísticas recomendadas pelas DCNs, torna-se necessário enfatizar que a educação como prática libertária sugerida por Paulo Freire, utilizada nas rodas de conversa, pode servir de alicerce para o desenvolvimento de competências e ações direcionadas à preparação de um egresso médico crítico, reflexivo, que estabeleça critérios embasados por responsabilidade social e cidadania (Freire, 1997).

A maioria dos entrevistados - 79,62\% - demonstrou interesse em participar de momentos educativos, enquanto aguarda por uma consulta. Segundo Aristóteles, “Todo homem deseja naturalmente saber”, além disso, alguns estudos comentam que nas salas de espera dos serviços de saúde, os clientes podem obter ambientes de acolhimento com ações de EPS, favorecendo os métodos de prevenção de doenças e de promoção da saúde, com contentamento diante dos benefícios obtidos. Para reforçar essa ideia, vale a pena comentar o resultado de um estudo realizado pela Escola de Farmácia e Odontologia de Alfenas, MG, em todas as clínicas de Odontopediatria do município mineiro, que demonstrou aprovação dos pais das crianças com consultas marcadas pelos serviços de saúde citados, ao serem questionados enquanto esperavam pelo atendimento, sobre a realização de momentos informativos de EPS naquele espaço (Freire, 1997).

No segundo momento da pesquisa, foi elaborada uma cartilha com a temática depressão, cujo conteúdo apresentou, em sua etapa de sistematização, uma revisão da literatura, em protocolos do Ministério da Saúde, para assegurar respaldo científico, para ser distribuída nos momentos dialógicos no ASB, a qual foi escrita na forma de sete perguntas e repostas contendo informações quanto ao conceito, causas, sintomas, riscos e tratamento da depressão. 
Os pontos positivos e negativos das rodas de conversa identificados pelos pacientes estão apresentados no Quadro 2.

Quadro 2 - Aspectos positivos e negativos das rodas de conversa identificados pelos pacientes.

\begin{tabular}{|c|c|}
\hline Variável & $\begin{array}{c}\text { Tabela 2 - Pontos positivos e negativos das rodas de conversa realizadas no ambulatório } \\
\text { Sette de Barros, no município de Ponte Nova, Minas Gerais. }\end{array}$ \\
\hline \multirow{3}{*}{$\begin{array}{c}\text { Trocas de experiências }-22^{\mathrm{x}} \\
\text { Pontos } \\
\text { positivos }\end{array}$} & Fácil entendimento $-56^{\mathrm{x}}$ \\
& Respeito $-13^{\mathrm{x}}$ \\
& Tempo muito curto $-0^{\mathrm{x}}$ \\
& Não tiveram pontos positivos $-0^{\mathrm{x}}$ \\
& Outros $-1^{\mathrm{x}}$ \\
\hline \multirow{3}{*}{ Pontos } & Ruídos no local $-0^{\mathrm{x}}$ \\
negativos & Muita mobilização de pessoas $-34^{\mathrm{x}}$ \\
& Não gostariam de ter participado $-0^{\mathrm{x}}$ \\
& Tempo muito curto $-58^{\mathrm{x}}$ \\
& Não tiveram pontos negativos $-2^{\mathrm{x}}$ \\
& Outros - $0^{\mathrm{x}}$ \\
\hline
\end{tabular}

${ }^{x}$ Número de respostas dadas pelas pessoas que responderam aos questionários. Fonte: Autores (2021).

Após a realização das rodas de conversa e a partir da aplicação do segundo questionário, verificou-se que todos os participantes da pesquisa ficaram confortáveis durante o momento dialógico e que a maioria teve a oportunidade de esclarecer suas dúvidas. Outro ponto positivo assinalado pelos pacientes foi o fácil entendimento - 56 pessoas -, o que possibilita concluir que os graduandos desenvolveram boas habilidades de comunicação, contribuindo para a formação de uma relação médicopaciente produtiva (Brasil, 2014) e, demonstrando humanização na interação entre os acadêmicos da FADIP e a clientela participante da roda de conversa. Isso, porque a humanização da Medicina compreende uma série de fatores voltados a uma boa relação médico-paciente, com atenção direcionada à percepção social e psicológica - que é sempre exclusiva - das consequências da presença de um determinado agravo de saúde, sobre determinado ser humano (Freire, 1997).

Ademais, enquanto ocorria a roda de conversa, o ponto negativo do momento dialógico referido pela maioria foi o tempo muito curto. Ao serem indagados quanto ao autocuidado, todos os entrevistados responderam que a roda de conversa contribuiu para o melhor entendimento de como cuidar de sua própria saúde, ou seja, a educação em saúde contribuiu para o autocuidado, demonstrando melhoria na qualidade dos serviços prestados embasados pela integralidade e bioética (Brasil, 2014).

Os pacientes foram tratados de forma humanizada pelos discentes com os quais dialogaram, refletindo em uma satisfação por parte dos entrevistados. O Ministério da Saúde, desde 2003, através da Política Nacional de Humanização da Atenção e Gestão do SUS (HumanizaSUS), prioriza um processo de trabalho em saúde com consideração e respeito aos distintos atores envolvidos na produção de saúde, sejam pacientes, profissionais ou gestores; aperfeiçoamento da autonomia intelectual frente aos processos decisórios em saúde, tanto a nível individual, quanto coletivo; possibilitar ao usuário que apresente condições para o seu autocuidado; o controle social na formulação de políticas públicas que se integram no SUS; levantamento dos principais agravos de saúde da comunidade; defesa da universalidade do acesso no SUS; valorização do cuidado interdisciplinar e multiprofissional; valorização do acolhimento, com redução das filas de espera por ações em saúde e maior resolutividade; melhora dos ambientes de trabalho e de atendimento; permitir o contato dos centros de graduação em saúde e escolas técnicas com o cenários práticos dos serviços de saúde; integralidade da atenção (Brasil, 2010). Essas características da humanização, somadas aos fatores bioéticos, se reforçam nas DCNs (Brasil, 2014).

Após a análise dos resultados, observou-se que a integração das políticas públicas Programa de Saúde da família e PNEPS-SUS, além das políticas de participação e controle social e iniciativas de participação popular, demonstrou que os atributos e funções essenciais da APS de foco na família e orientação comunitária, que correspondem às bases para o 
planejamento e a ação em saúde do território foram alcançados com as rodas de conversa, uma vez que os usuários elegeram a temática a ser debatida e aprovaram a metodologia de abordagem do assunto. Além disso, a proposta do presente estudo apresentou pontos positivos para a formação dos graduandos de Medicina da FADIP, os quais atuaram como participantes das rodas de conversa, orientando o diálogo e também distribuindo as cartilhas com a temática depressão, porque conseguiram, por meio de metodologias ativas de ensino e aprendizagem, realizadas no ASB, experienciar os três eixos sugeridos pela DCNs: atenção à saúde, gestão em saúde e educação em saúde (Brasil, 2014).

\section{Considerações Finais}

A experiência mostrou aproveitamento mútuo para discentes e população, propiciando a integração de políticas públicas no SUS, através da Estratégia de Saúde da Família e a Política Nacional de Educação Popular em Saúde, por meio da realização de rodas de conversa, na sala de espera do ASB. Tal experiência possibilitou fortalecer a tríade ensino-serviço-pesquisa.

Após análise dos dados do questionário, foi verificado que as palestras dialogadas realizadas promoveram satisfação da clientela participante do momento educativo. Adicionalmente houve possibilidade de construção do vínculo médico-paciente, com conhecimento partilhado entre os dois sentidos da relação. A elaboração da cartilha permitiu o compartilhamento de informações possibilitando maior poder de decisão do usuário frente aos fatores causais, sintomatológicos, diagnósticos, terapêuticos e prognósticos da depressão, valorizando a participação democrática dos cidadãos nas ações da política pública de saúde.

Conclui-se que a participação dos usuários do SUS na construção dos momentos educativos, permite que eles se sintam como parte do processo, através do espaço acolhedor e, concomitantemente, crítico-reflexivo. Assim, ocorre valorização do paciente e oportunidade de crescimento aos alunos e a educação popular em saúde acontece de forma direcionada à legitimação e ao fomento de políticas públicas na saúde, além de promover a autonomia intelectual dos sujeitos, proporcionando melhoria no autocuidado e aprendizado.

\section{Referências}

Adler, M. S. \& Gallian, D. M. C. (2014). Formação médica e serviço único de saúde: propostas e práticas descritas na literatura especializada. Revista Brasileira de Educação Médica, 38(3), 388-396.

Bordenave, J. E. D. (1999). Alguns fatores pedagógicos. In: Santana, J. P., Castro, J. L., organizadores. Capacitação em Desenvolvimento de Recursos Humanos - CADRHU (pp.261-268). Ministério da Saúde/Organização Pan-Americana da Saúde/ Editora da UFRN.

Brasil. DATASUS CNES. (2015). http://datasus.saude.gov.br/cadastro-nacional-de-estabelecimentos-de-saude.

Brasil. Ministério da saúde. Política Nacional de Educação Popular em Saúde no Sistema Único de Saúde. (2013). Ministério da Saúde. https://bvsms.saude.gov.br/bvs/saudelegis/gm/2013/prt2761_19_11_2013.html

Brasil. Resolução CNE/CES 3/2014. (2014). Diário Oficial da União, Brasília: Ministério da Saúde/ Ministério da Educação.: http://portal.mec.gov.br/conaescomissao-nacional-de-avaliacao-da-educacao-superior/323-secretarias-112877938/orgaos-vinculados-82187207/20138-ces-2014

Costa, D. A. S., Silva, R. F. D., Lima, V. V. \& Ribeiro, E. C. O. (2018). Diretrizes curriculares nacionais das profissões da Saúde 2001-2004: análise à luz das teorias de desenvolvimento curricular. Interface-Comunicação, Saúde, Educação, 22(67), 1183-95.

Echer, I. C. (2005). Elaboração de manuais de orientação para o cuidado em saúde. Revista Latino-Americana de Enfermagem, $13(5)$, 754-757.

Ferreira, L., Barbosa, J. S. D. A., Esposti, C. D. D. \& Cruz, M. M. D. (2019). Educação Permanente em Saúde na atenção primária: uma revisão integrativa da literatura. Saúde em Debate, 43(120), 223-239.

Ferreira, M. J. M., Ribeiro, K. G., Almeida, M. M. D., Sousa, M. D. S. D., Ribeiro, M. T. A. M., Machado, M. M. T., \& Kerr, L. R. F. S. (2019). New National Curricular Guidelines of medical courses: opportunities to resignify education. Interface-Comunicação, Saúde, Educação, 23(1), e170920.

Ferreira, V. F., Rocha, G. O. R. D., Lopes, M. M. B., Santos, M. S. D., \& Miranda, S. A. D. (2014). Educação em saúde e cidadania: revisão integrativa. Trabalho, educação e saúde, 12(2), 363-378.

Freire, P. (1999). Educação como prática da liberdade. Paz e Terra. 
Research, Society and Development, v. 10, n. 13, e456101321256, 2021

(CC BY 4.0) | ISSN 2525-3409 | DOI: http://dx.doi.org/10.33448/rsd-v10i13.21256

Freire, P. (1997). Pedagogia da autonomia: saberes necessários à prática educativa. Paz e Terra.

França, R. R. D. \& Maknamara, M. (2019). A literatura sobre metodologias ativas em educação médica no Brasil: notas para uma reflexão crítica. Trabalho, educação e saúde, 17(1), 1-22.

Morin, E. (2003). A cabeça bem-feita: repensar a reforma, reformar o pensamento (8a ed.), Bertrand Brasil.

Nascimento, A. K. C., \& Baduy, R. S (2021). Simulação, oficina e roda de conversa: estratégias de aprendizagem ativa na saúde. Revista Educação em Debate. 43(84), 152-167.

Oliveira, L. M. P. \& Leite, M. T. M. (2011). Concepções Pedagógicas. Módulo Pedagógico. Especialização em Saúde da Família - Modalidade a Distância. UNA-SUS UNIFESP. https://www.unasus.unifesp.br/biblioteca_virtual/esf/1/modulo_pedagogico/Unidade_1.pdf

Oliveira, M. F. \& Cota, L. G. S. (2018). A pedagogia freiriana nas práticas de educação em saúde. Diversitates International Journal, 10(1), 46-58.

Paro, C. A., Ventura, M. \& Silva, N. E. K. (2019). Paulo Freire e o inédito viável: esperança, utopia e transformação na saúde. Trabalho, Educação e Saúde, 18(1), 1-22.

Pellegrini Filho, A., Buss, P. M. \& Esperidião, M. A. (2013). Promoção da Saúde e seus fundamentos: determinantes sociais da saúde, ação intersetorial e políticas saudáveis. In: Paim, J. S., Almeida Filho, N. Saúde coletiva: teoria e prática (pp. 305-326). Med Book.

Pereira, A. S. et al. (2018). Metodologia da pesquisa científica. Biblioteca Central da UFSM. https://repositorio.ufsm.br/bitstream/handle/1/15824/Lic_C omputacao_Metodologia-Pesquisa-Cientifica.pdf?sequence=1

Silva, C. B. G. \& Scherer, M. D. D. A. (2020). A implementação da Política Nacional de Educação Permanente em Saúde na visão de atores que a constroem. Interface-Comunicação, Saúde, Educação, 24 (19), e190840.

Starfield, B. (2002). Atenção primária: equilíbrio entre necessidades de saúde, serviços e tecnologia. UNESCO, Ministério da Saúde. 